\title{
La tecnología DGPS en la construcción: control de movimientos en grandes estructuras
}

\author{
DGPS technology in construction: movement control in \\ large structures
}

\author{
M. Marchamalo ${ }^{(*)}$, D. Galán, J. A. Sánchez, R. Martínez
}

\section{RESUMEN}

La evolución conjunta de las tecnologías de auscultación y las telecomunicaciones está permitiendo el desarrollo de Sistemas de Auscultación Automática de estructuras. En este trabajo se analiza la viabilidad de la aplicación de la técnica DGPS a la auscultación en construcción. Se puede afirmar que el GPS diferencial es preciso y útil en la auscultación de grandes estructuras, como las presas, al reflejar adecuadamente el movimiento de las mismas, en comparación con los sensores geotécnicos. El empleo de DGPS en grandes estructuras puede proporcionar información sobre sus movimientos y deformaciones. Esta información puede estar disponible en tiempo real, con sistemas automáticos de medición y alarmas programadas para distintos umbrales de movimientos. Por todo ello, se considera que el DGPS es una tecnología complementaria para la auscultación de grandes estructuras, que puede integrarse con otros métodos geotécnicos o topográficos, tanto en la fase constructiva como en el seguimiento de las obras.

479-2

Palabras clave: auscultación, DGPS, grandes estructuras, presas, tiempo real

\section{SUMMARY}

The joint development of auscultation and communications technologies is enabling the development of Automated Monitoring Systems for structures. In this paper, we analyze the viability of the application of DGPS (Differential GPS) technique to monitoring in construction. We can affirm DGPS is accurate and useful for the auscultation of large structures, such as dams, reflecting properly their movements and deformations, compared to geotechnical sensors. The use of DGPS technology in large structures can provide information on the dynamic characteristics of their movements and deformations. All this can be analyzed in real time, with automated measurement systems and alarms programmed for different thresholds. Therefore DGPS is considered a complementary technique for auscultation of large structures that can integrate with other geotechnical or topographical methods, both in the construction and monitoring phases.

Keywords: auscultation, DGPS, large structures, dams, real time

(*) E.T.S.I. Caminos, Canales y Puertos. Universidad Politécnica de Madrid, (España) 


\section{CONTROL DE MOVIMIENTOS Y DEFORMACIONES EN LA CONSTRUCCIÓN}

El control de movimientos es un aspecto fundamental en la fase constructiva y en la fase de seguimiento post-construcción (1). En la construcción puede interesar controlar los movimientos de las zonas en consolidación (estructuras de tierra, taludes, escombreras,...), de los propios edificios e infraestructuras o partes de los mismos y de las zonas aledañas en las que se puedan producir algunos efectos inducidos por el proceso constructivo.

Entre los métodos de control de movimientos en estructuras se encuentran los topográficos clásicos y los geotécnicos. Los métodos topográficos clásicos más frecuentes son nivelación de alta precisión, trilateración, triangulación, poligonación de precisión, colimación angular, etc..., realizadas con personal especializado e in situ. Los métodos geotécnicos se basan en instrumentos que miden desplazamientos relativos o variables ambientales relevantes como los péndulos, inclinómetros, girómetros, piezómetros,....

Con los nuevos avances tecnológicos, se han ido añadiendo otro tipo de métodos al control de deformaciones y de movimientos, como los basados en fibra óptica, estaciones robotizadas de topografía o mediante GPS diferencial Differential Global Positioning System). La conjunción de los avances tecnológicos en medición y comunicaciones ha permitido el desarrollo de Sistemas de Auscultación Automática, que permiten la automatización de la medición, el control continuo de las deformaciones y garantizar la uniformidad y calidad de las mediciones, al reducir el error de origen humano. Olalla (2007) analizó las principales técnicas tradicionales de control que se han utilizado en auscultación, indicando sus umbrales de precisión (2). Se ha completado esta información en la Tabla 1, que presenta los principales métodos de auscultación y sus características:

La tecnología GPS diferencial o DGPS permite la auscultación con coordenadas absolutas (geodésicas) en continuo y con alarmas en tiempo real. Para su aplicación, la única limitación es que los puntos de control han de tener horizonte despejado y ausencia de elementos cercanos que puedan causar multitrayectoria. La tecnología DGPS se ha aplicado en geología principalmente en estabilidad de laderas y terraplenes, donde la precisión requerida es centimétrica.

El avance de la tecnología y las comunicaciones de los últimos años, ha permitido desa-

Tabla 1

Técnicas de control y auscultación

\begin{tabular}{|c|c|c|c|c|}
\hline Tipo & Técnica de control & Automática & $\begin{array}{c}\text { Frecuencia } \\
\text { medición }(\mathrm{Hz})\end{array}$ & $\begin{array}{l}\text { Límite } \\
\text { de precisión }\end{array}$ \\
\hline TOPOGRAFÍA CLÁSICA & Fotogrametría & $\mathrm{NO}$ & $<10^{-4}$ & Según escala (2) \\
\hline TOPOGRAFÍA CLÁSICA & Topografía con nivel & $\mathrm{NO}$ & $<10^{-4}$ & $1 \mathrm{~mm}$ \\
\hline $\begin{array}{c}\text { TOPOGRAFÍA CLÁSICA } \\
\text { GEODÉSICA }\end{array}$ & Topografía con estación total & $\mathrm{SI}$ & $<0,3$ & $\pm 0,6 \mathrm{~mm}+1 \mathrm{ppm}(3)$ \\
\hline TOPOGRAFÍA GEODÉSICA & GPS diferencial & $\mathrm{SI}$ & $10-100$ & $\begin{array}{l}\mathrm{V}: 20 \mathrm{~mm} \mathrm{H}: 10 \mathrm{~mm} \\
(3 \mathrm{~mm} \text { con proceso) }(3)\end{array}$ \\
\hline $\begin{array}{c}\text { TOPOGRAFÍA CLÁSICA } \\
\text { GEODÉSICA }\end{array}$ & RADAR base (GBSAR) & $\mathrm{SI}$ & $<0,001$ & $1-5 \mathrm{~mm}(4)$ \\
\hline $\begin{array}{c}\text { TOPOGRAFÍA CLÁSICA/ } \\
\text { GEODÉSICA }\end{array}$ & Láser terrestre & $\mathrm{SI}$ & $<1$ & $1-5 \mathrm{~mm}(3)$ \\
\hline GEOTÉCNICA & Inclinometría & $\mathrm{SI}$ & $1-10$ & $0,1 \mathrm{~mm}(2)$ \\
\hline GEOTÉCNICA & Girómetros en superficie & SI & $1-10$ & $0,05 \mathrm{~mm} / \mathrm{m}(2)$ \\
\hline GEOTÉCNICA & Extensómetros / Fisurómetros & $\mathrm{SI}$ & $1-10$ & $\begin{array}{c}\mathrm{V}: 0,001 \mathrm{~mm} \\
\mathrm{H}: 0,01 \mathrm{~mm}(2)\end{array}$ \\
\hline GEOTÉCNICA & Piezometría & SI & $<1$ & Variable (2) \\
\hline GEOTÉCNICA /AMBIENTAL & $\begin{array}{c}\text { Controles meteorológicos } \\
\text { (temperatura, viento, precip.) }\end{array}$ & $\mathrm{SI}$ & $<1$ & Variable \\
\hline
\end{tabular}


rrollar métodos que proporcionan posiciones con exactitud milimétrica ampliando sus aplicaciones hasta el control de estructuras.

Galán et al. (5) realizaron una revisión de literatura sobre el empleo mundial de la técnica DGPS en la última década, que se ha concentrado principalmente en el control de movimientos en presas, puentes, torres, estructuras de tierra y laderas. Según estos autores, China y EEUU son los países donde más se ha desarrollado la investigación de la aplicación de esta técnica. China ocupa el primer lugar tanto en el control de movimientos de puentes, rascacielos e infraestructuras, como también en diversas áreas como el control de subsidencias en el terreno.

La auscultación de grandes estructuras se enmarca en el seguimiento de su salud estructural (Global Navigation Satellite System) y presenta particularidades especiales, debido fundamentalmente a la naturaleza y dinámica de las mismas (6). Cheng et al. (7) realizaron una revisión de las experiencias en monitorización dinámica de grandes estructuras con tecnología GPS, citando varias experiencias satisfactorias en puentes, torres, rascacielos y estructuras de gran esbeltez y flexibilidad. Según señalan Psimoulis et al. (8), el GPS puede además proporcionar información sobre la vibración y las frecuencias características de grandes estructuras flexibles con gran precisión, resolviendo parte de los problemas que presentan los acelerómetros, como son la propagación de errores y su dificultad para detectar desplazamientos semi-estáticos y vibraciones de baja frecuencia, característicos de las grandes estructuras flexibles sometidas a acciones como el viento.

Este trabajo presenta el estado de la técnica DGPS en la construcción y analiza su viabilidad para el control de movimientos de estructuras. Se exponen datos del estudio de caso de la presa de "La Aceña" (Ávila). Ésta presa es arco-gravedad de $68 \mathrm{~m}$ de altura sobre cimientos, con gran esbeltez y gran amplitud de movimientos en coronación analizados y conocidos desde el año 1990, y se está controlando con DGPS, dentro de la línea de investigación realizada en el Laboratorio de Topografía y Geomática de la E.T.S.I. de Caminos, Canales y Puertos de Madrid sobre DGPS aplicado a la ingeniería civil.

\section{LA TECNOLOGÍA GPS Y GPS DIFERENCIAL (DGPS)}

La tecnología GPS ha evolucionado mucho recientemente, pasando de las aplicaciones militares en el siglo XX a ser una de las principales herramientas del desarrollo tecnológico en el siglo XXI. En la actualidad los sistemas de posicionamiento por satélite evolucionan hacia la integración de diferentes constelaciones, tanto la del GPS (NAVSTAR), como la rusa GLONASS y la futura europea Galileo, lo que se denomina GNSS (Global Navigation Satellite System). La integración actual de datos de NAVSTAR (EEUU) y GLONASS (Rusia), permite ampliar el rango de empleo de la técnica y su precisión en condiciones de baja visibilidad de bóveda celeste, como son los entornos urbanos, forestales y con pantallas laterales.

La técnica DGPS se basa en el empleo conjunto de dos tipos de estaciones GPS: referencias o fijas y móviles, situadas lo suficientemente cerca entre sí como para asegurar que la perturbación atmosférica de la señal recibida en ambos puntos sea similar. De esta manera, la posición de cada punto móvil se refiere continuamente a la de la base fija o de referencia, constituyendo una líneabase monitorizada continuamente. Las estaciones fijas o de referencia, cuyas coordenadas son conocidas, permiten postprocesar en continuo las posiciones de los puntos móviles, obteniendo precisiones de orden centimétrico $(\mathrm{V}: 20$ $\mathrm{mm} \mathrm{H}$ : $10 \mathrm{~mm}, 3 \mathrm{~mm}$ con postproceso, según (3)). El filtrado matemático de los datos permite ajustar las posiciones a la velocidad observada de movimiento, en función de las posiciones anteriores, permitiendo alcanzar una exactitud milimétrica en oscilaciones de baja frecuencia.

\subsection{Bases de la técnica DGPS}

Los sistemas GPS cuentan con un segmento espacial (satélites), terrestre (estaciones de control) y de usuario (receptores). Los receptores GPS realizan dos clases de medidas a partir de las ondas recibidas de los satélites: pseudodistancias de código (C/A en L1 y L2C y P en L1 y L2) y medidas de fase de la portadora (en L1 y L2). La pseudodistancia es la medida de la distancia entre satélite y receptor, obtenida a partir del tiempo de transmisión de la señal multiplicado por la velocidad de la luz.

El tiempo se obtiene mediante la correlación o alineamiento del código PRN (pseudo random noise) generado por el receptor y el idéntico emitido por el satélite. Prescindiendo de los errores propios en GPS (estados de reloj, órbitas, troposfera, ionosfera, "multipath" o multritrayectoria, etc), con los códigos se obtienen precisiones nominales en las pseudodistancias del $1 \%$ de la longitud del chip (cada cuánto se repite el código), resultando $3 \mathrm{~m}$ para el código C/A y 0,3 m para el código $\mathrm{P}$.

El observable de fase es la diferencia entre la fase de la portadora recibida del satélite 
y la fase generada internamente por el oscilador del receptor. Estas medidas de fase se registran en intervalos iguales de tiempos del receptor, y no tienen en cuenta el número de ondas enteras que hay entre el receptor y el satélite.

La pseudodistancia que se obtiene se deriva de una medida de la fase de la portadora en vez de derivarse a partir de una medida del tiempo. La distancia satélite-receptor en el instante inicial $t_{0}=0$ está relacionada con el número entero de longitudes de onda $(T)$ y su fase $(\Delta \varphi)$ (parte fraccional de longitud de onda) siendo $f$ la frecuencia de la onda portadora [1]:

$$
D=\frac{c \cdot \Delta \varphi}{f}+\lambda \cdot N
$$

A medida que el satélite se aleja o se acerca del receptor, éste va generando ciclos enteros de onda para seguir la señal del satélite, de tal forma que el receptor mide una parte fraccional de la fase $\left(\varphi_{R}^{S}\right)$ y el número entero de longitudes de onda que se acerca/aleja el satélite $\left(n_{R}^{S}\right)$. El número entero de longitudes de onda inicial entre satélite y receptor $\left(N_{R}^{S}\right)$, no se puede medir, es la principal incógnita a determinar en un cálculo GPS y es lo que se conoce como ambigüedad de ciclo inicial, de tal forma que la medida de la pseudodistancia $\left(\rho_{R}^{S}\right)$ con medidas de fase (expresada en ciclos) será [2]:

$\rho_{R}^{S}\left(\frac{1}{\lambda}\right)=\varphi_{R}^{S}+n_{R}^{S}+N_{R}^{S}$

Nominalmente, la precisión con que se puede medir la fase en un receptor es de 0,01 ciclos, lo cual implica que para las longitudes de onda en las portadoras GPS (unos 19 $\mathrm{cm}$ para L1 y $24 \mathrm{~cm}$ para L2), la medida de pseudodistancias de fase sin tener en cuenta los errores propios del sistema anteriormente citados, puede tener una precisión del orden de $2 \mathrm{~mm}$.

El método relativo o diferencial GPS de observación simultánea de la misma constelación de satélites entre dos puntos (denominados referencia o base y el otro "rover" o móvil), elimina la mayor parte de los errores que afectan al GPS, siendo éste, evidentemente, el que se utiliza en posicionamiento de precisión.

La precisión del método relativo o diferencial GPS con medidas de fase ha demostrado que se pueden alcanzar precisiones del orden milimétrico, dependiendo sobre todo de la distancia de líneabase y de la combinación de observables que se utilice. En función de la distancia de líneabase, también es importante el método que se utilice de resolución de ambigüedades, pero generalmente, con distancias tan cortas como son las del caso que nos ocupa, incluso con receptores GPS monofrecuencia L1, es factible alcanzar una precisión por debajo de los $5 \mathrm{~mm}$.

Si se denomina $\varphi$ a la parte que mide el receptor $(\varphi+N)$, dt al estado del reloj de satélite, dT al estado de reloj de receptor, $f$ frecuencia y $d_{\text {trop }}$ y $d_{\text {ion }}$ los efectos troposférico e ionosférico respectivamente de distinto signo, se puede generalizar la ecuación de fase observada por el receptor i del satélite $\mathrm{k}$ en el instante t como [3]:

$\Phi_{i}^{k}(t)=\frac{1}{\lambda} \rho_{i}^{k}(t)+f^{k}\left[d^{k}(t)-d T_{i}(t)\right]+$

$+N_{i}^{k}+\frac{1}{\lambda}\left[d_{\text {trop }}(t)-d_{\text {ion }}(t)\right]$

Las simples diferencias entre receptores se establecen a partir de la diferencia de fase obtenida mediante la observación simultánea de un satélite $k$ en dos estaciones i y j, de tal forma que, restando las ecuaciones de fase en ambas estaciones, resultará [4]:

$$
\begin{aligned}
& \Phi_{i, j}^{k}(t)=\frac{1}{\lambda} \rho_{i, j}^{k}(t)-f^{k} d T_{i, j}(t)+ \\
& +N_{i, j}^{k}-\frac{1}{\lambda} d_{i o n i, j}^{k}(t)+\frac{1}{\lambda} d_{\text {tropi }, j}^{k}(t)
\end{aligned}
$$

con lo cual, como se puede ver, se elimina el error en el oscilador del satélite con respecto al tiempo GPS y si las estaciones están próximas, lo mismo ocurrirá con los dos últimos términos de correcciones ionosféricas y troposféricas, que son, por otro lado, las más importantes en cualquier observación GPS.

En el modelo de dobles diferencias de fase es necesario considerar dos estaciones $(i, j)$ observando simultáneamente a dos satélites $(k, l)$, de tal forma que restando las ecuaciones de simples diferencias de fase anteriores en el mismo instante t, se obtendrá: [5]

$$
\begin{aligned}
& \Phi_{i, j}^{k, l}(t)=\frac{1}{\lambda} \rho_{i, j}^{k, l}(t)+N_{i, j}^{k, l}- \\
& -\frac{1}{\lambda} d_{i o n i, j}^{k, l}(t)+\frac{1}{\lambda} d_{t r o p i, j}^{k, l}(t)
\end{aligned}
$$

De esta forma se eliminan los errores de reloj de los receptores, aunque sigue persistiendo la incógnita de ambigüedad de ciclo inicial entre ambas estaciones. Análogamente las correcciones atmosféricas pueden ser eliminadas si se consideran iguales efectos en ambas estaciones debido a su proximidad. 
El modelo de posicionamiento que utilizan la mayor parte de los programas de auscultación es el de triples diferencias, el cual se basa en la diferencia de dobles diferencias entre dos estaciones y dos satélites y entre una época de observación y la siguiente, en dos instantes $\mathrm{t}_{1}$ y $\mathrm{t}_{2}[6]$ :

$$
\Phi_{i, j}^{k, l}\left(t_{2}\right)-\Phi_{i, j}^{k, l}\left(t_{1}\right)=\frac{1}{\lambda}\left[\rho_{i, j}^{k, l}\left(t_{2}\right)-\rho_{i, j}^{k, l}\left(t_{1}\right)\right]-\frac{1}{\lambda}\left[d_{i o n i, j}^{k, l}\left(t_{2}\right)-\right.
$$$$
\left.-d_{i o n i, j}^{k, l}\left(t_{1}\right)\right]+\frac{1}{\lambda}\left[d_{\text {tropi }, j}^{k, l}\left(t_{2}\right)-d_{t r o p i, j}^{k, l}\left(t_{1}\right)\right]
$$

Como se puede observar en este modelo, considerando que las ambigüedades se mantienen entre dos épocas consecutivas t1 y $\mathrm{t} 2$, la gran ventaja en las triples diferencias es que se eliminan las ambigüedades de ciclo inicial y además no está afectado por la pérdida de ciclos que se puede producir en cualquier receptor GPS debido a una baja relación señal-ruido.

Igualmente, si los puntos están próximos y las épocas consecutivas muy próximas, los errores ionosférico y troposférico pueden prácticamente eliminarse. La refracción troposférica normalmente no cambia rápidamente con el tiempo y es por tanto considerablemente reducida en las triples diferencias. No sucede lo mismo, sin embargo, para la refracción ionosférica, la cual puede variar rápidamente con el tiempo, sobre todo en zonas de latitud extrema.

La combinación libre ionosfera con observables de fase L1 y L2 se usa para eliminar el efecto ionosférico. En el caso del control de deformaciones en estructuras, las estaciones referencia y móviles suelen estar situadas a distancias no superiores al kilómetro, con lo cual no es imprescindible el disponer de receptores de doble frecuencia, con el consiguiente ahorro económico en el equipamiento.

La resolución de las lineabase con triples diferencias se hace en tiempo real con la transmisión de observables desde tres receptores al centro de control y el software aplica el filtro Kalman (con parámetros configurables según el tipo de movimiento esperable) para el suavizado de los resultados, permitiendo de esta forma precisiones en torno a 1 milímetro.

\subsection{Filtrado de datos}

El filtrado de datos tiene por objeto mejorar la exactitud de la solución de posición, estableciendo un algoritmo de tendencia en base a los datos observados. El filtro más empleado para este tipo de datos es el filtro propuesto por Kalman (9). El filtro Kalman está constituido por un conjunto de ecuaciones matemáticas que proporcionan una estimación recursiva (por iteración) del estado de un sistema (en este caso una posición), minimizando el error cometido. El filtro permite estimar y/o predecir el estado de un sistema en el pasado, presente y futuro, aún cuando la naturaleza precisa del mismo es desconocida, (10).

De forma análoga, el sistema de control de movimientos basado en la tecnología DGPS puede combinar medidas de alta sensibilidad en tiempo real con estabilidad a largo plazo implementando un filtro Kalman, pudiéndose alcanzar sensibilidades en el sistema de hasta $\pm 1 \mathrm{~mm}$. Esta exactitud es la que le puede hacer muy útil y competitivo en el control de movimientos de infraestructuras con ciclos de movimientos radiales y tangenciales que siguen velocidades y pendientes de deformación conocidas. Esto favorece el mejor ajuste del filtro y reduce el error de la posición filtrada con respecto a la posición real.

El filtro es un procedimiento matemático que opera por medio de un mecanismo de predicción y corrección. En esencia este algoritmo pronostica el nuevo estado a partir de su estimación previa añadiendo un término de corrección proporcional al error de predicción, de tal forma que este último es minimizado estadísticamente.

Desde este punto de vista las ecuaciones que se utilizan para derivar el filtro de Kalman se pueden dividir en dos grupos:

- Las que actualizan el tiempo o ecuaciones de predicción.

- Las que actualizan los datos observados o ecuaciones de actualización.

Las del primer grupo son responsables de la proyección del estado al momento t tomando como referencia el estado en el momento t-1 y de la actualización intermedia de la matriz de covarianza del estado. El segundo grupo de ecuaciones son responsables de la retroalimentación, es decir, incorporan nueva información dentro de la estimación anterior con lo cual se llega a una estimación mejorada del estado.

Las ecuaciones específicas para el pronóstico y la corrección del estado son [7],[8] $(10,11)$ :

$\hat{X}_{t}^{*}=A \cdot \hat{X}_{t-1}$

$P_{t}^{*}=A \cdot P_{t-1} \cdot A^{T}+Q$

$X^{*}$ : estimación del estado del sistema

$\mathrm{X}$ : estado del sistema en un cierto tiempo

A: matriz de cambio entre estados

$\mathrm{P}$ : covarianza del residuo en un cierto tiempo

$P^{*}$ : estimación de la covarianza

Q: covarianza de la perturbación aleatoria 
del proceso que trata de estimar el estado, [9],[10],[11].

$$
\begin{aligned}
& K_{t}=P_{t}^{*} \cdot H^{T} \cdot\left(H \cdot P_{t}^{*} \cdot H^{T}+R\right)^{-1} \\
& \hat{X}_{t}=\hat{X}_{t}^{*}+K_{t} \cdot\left(Z_{t}-H \cdot \hat{X}_{t}^{*}\right) \\
& P_{t}=\left(I-K_{t} \cdot H\right) \cdot P_{t}^{*}
\end{aligned}
$$

$Z_{t}$ : medida obtenida del estado del sistema

$\mathrm{H}$ : matriz que relaciona el estado del sistema con la medida obtenida del mismo

$\mathrm{K}_{\mathrm{t}}$ : matriz de ganancia o factor de ganancia Kalman, que minimiza la covarianza del residuo calculada o a posteriori $\left(\mathrm{P}^{*}\right)$

$\left(\mathrm{Z}_{\mathrm{t}}-\mathrm{H} \cdot \mathrm{X}_{\mathrm{t}}\right)$ es el residuo o discrepancia entre la medida predicha y la medición real.

La primera tarea durante la corrección de la proyección del estado es el cálculo de la ganancia de Kalman, $K_{t}$ [9]. Este factor de ponderación o ganancia es seleccionado de tal forma que minimice la covarianza del residuo de la nueva estimación del estado. El siguiente paso es medir el proceso de forma real para obtener $Z_{t} y$ entonces generar una nueva estimación del estado que incorpora la nueva observación como en la ecuación [10]. El paso final es obtener una nueva estimación de la covarianza del error mediante la ecuación [11]. Después de cada par de actualizaciones, tanto del tiempo como de la medida, el proceso es repetido tomando como punto de partida las nuevas estimaciones del estado y de la covarianza del residuo (11). Esta naturaleza recursiva es una de las características Ilamativas del filtro de Kalman que permiten o facilitan su programación.

Hofmann-Wellenhof en su libro "GPS Theory and Practice" en lo que se refiere a la aplicación del filtrado Kalman, asemeja cualquier sistema dinámico de control de movimientos mediante DGPS, a un vehículo en movimiento, donde las coordenadas y la velocidad forman parte del vector de estado que se puede predecir en cualquier tiempo $\mathrm{t}$, en base al sistema de ecuaciones aquí descrito (12).

\subsection{Aplicaciones DGPS}

Las principales aplicaciones del DPGS al control de movimientos son (5):

- Estructuras: Puentes, presas y diques de control, torres y rascacielos, plataformas petrolíferas, tuberías...
- Fenómenos naturales: Deslizamientos de laderas y subsidencias del terreno, terremotos, volcanes,...

Los principales programas de auscultación con técnicas DGPS son:

- 3D TRACKER, desarrollado por Condor Earth Technologies Inc. y representado por Pinnacle y actualmente Applied Geomechanics. Permite realizar seguimiento 3D en tiempo real de grandes estructuras, con resolución de triples diferencias y filtrado de Kalman. Ha sido empleado en España en la Aceña (5).

- GOCA (GPS-based Online Control and Alarm), desarrollado en la Universidad de Karlsruhe (Kalber et al., 2000) para centralizar la solución de red para baselíneas de receptores ubicados en puntos móviles a lo largo de un período de tiempo. En la actualidad está representado por Topcon Europa (13).

- INTETRAK, desarrollado por Orion Monitoring Systems Inc. para el seguimiento de desplazamientos en tiempo real y la integración de sistemas GPS (14).

- RTK Extend ${ }^{\mathrm{TM}}$. Desarrollado por NavCom Technologies Inc. (NavCom, 2006).

-SPIDER, desarrollado por Leica Geosystems, que permite la integración de redes de estaciones GPS. Ha sido empleado en numerosos estudios de caso en el mundo (3).

-4D Control de Trimble, aplicado en la presa de Tolt (Seattle, EEUU).

- Geo++ Gmbh ofrece un módulo de control de deformaciones en tiempo real con aplicaciones en monitorización de estructuras hidráulicas (15).

El control de movimientos en torres y rascacielos tiene similitudes con el de las presas destacando los artículos "Large Structure Health Dynamic Monitoring Using GPS Technology" (Cheng et al, 2002, China (7)) y "GPS in dinamic monitoring of longperiod structures" (Çelebi, 2000, EEUU, (16)) en los que se presentan diferentes casos monitorizados de desplazamientos instantáneos y a largo plazo bajo el efecto de algún sismo.

En la literatura se recogen algunos casos de implantación de los sistemas DGPS telecontrolados en tiempo real en las presas de $\mathrm{Pa}$ coima (EEUU) (14) y Libby (Montana, EEUU) (17), situadas en California y Montana, cuya configuración ha servido de base para el estudio de caso de la presa de "La Aceña" 
(5). Otra experiencia a nivel europeo en la auscultación mediante DGPS, es la presa de Kops (Austria). Esta presa cuenta con una antena de referencia y otras dos colocadas en coronación (GPS-based online Control and Alarm System) (13).

En cuanto al control de deformaciones y movimientos de puentes hay abundantes referencias, en la mayor parte de los casos sobre la puesta en carga y al control de las flechas ante solicitaciones instantáneas como los terremotos. Hay varias experiencias en literatura sobre el control de movimientos en laderas. Una aplicación en España es el control del movimiento de la ladera del Embalse de Arenós (18). bases de lectura inferiores en la galería perimetral, (Figura 2).

Existen bases de lectura en la intersección de cada péndulo con cada una de las galerías. En todas las bases de la galería perimetral y en la base del péndulo 2 de la

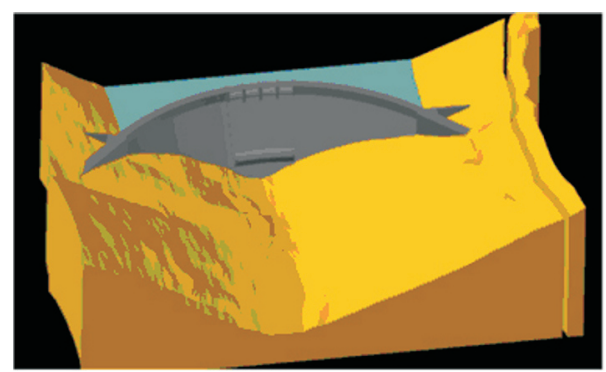

1. Vista 3D de la presa de La Aceña. Ávila (España). Fuente: (19).

2. Localización de los péndulos en alzado en la presa de la Aceña. Fuente: (19)

3. Movimientos radiales de los péndulos de la Presa de La Aceña. Informe anual de Auscultación 2008 (19)

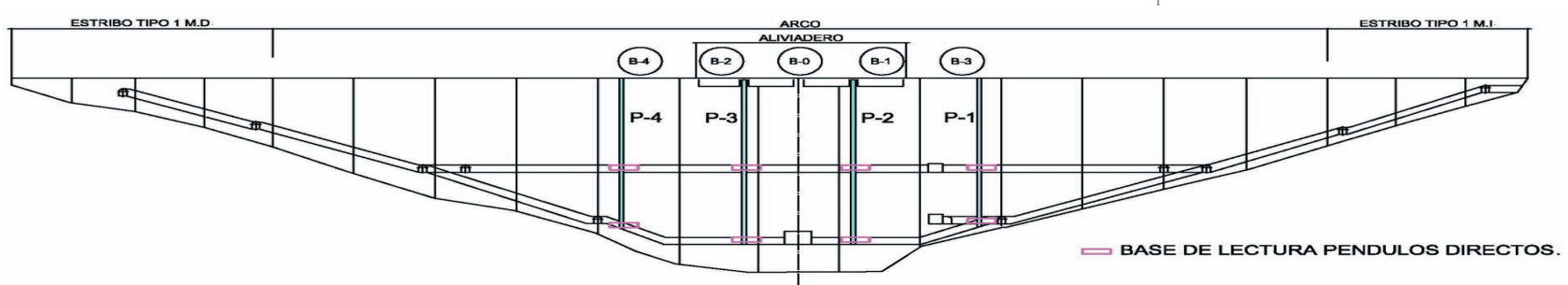

La conclusión más clara, obtenida en este análisis, es que se alcanzan las precisiones requeridas, siendo más tolerantes en los campos de la geología y estructuras de tierra y menos en el control de infraestructuras de hormigón, donde se requiere llegar a niveles milimétricos.

\section{APLICACIÓN DE LA TÉCNICA DGPS A UNA PRESA ARCO-GRAVEDAD EN ESPAÑA}

La presa de "La Aceña" está situada en la cuenca del río Aceña (perteneciente a la Cuenca del Tajo), en el término municipal de Peguerinos, provincia de Ávila (Comunidad Autónoma de Castilla-León, (Figura 1).

La presa es de hormigón, del tipo arco gravedad y planta curva con $68 \mathrm{~m}$ de altura sobre cimientos, con una gran esbeltez que genera una amplitud de movimientos radiales de ciclo anual, debida a las solicitaciones externas de carga de agua y temperatura, del orden de 35-40 mm (19).

El paramento de aguas arriba tiene talud vertical y el de aguas abajo 0,4. La coronación está situada a la cota $1.319 \mathrm{~m}$. Tiene una longitud de $340 \mathrm{~m}$ y un ancho de $6 \mathrm{~m}$, con una calzada de $4,5 \mathrm{~m}$ y 2 aceras de $0,75 \mathrm{~m}$

La presa dispone de cuatro péndulos, todos ellos directos, que dan información de los desplazamientos en coronación respecto de los puntos en que están ubicadas las
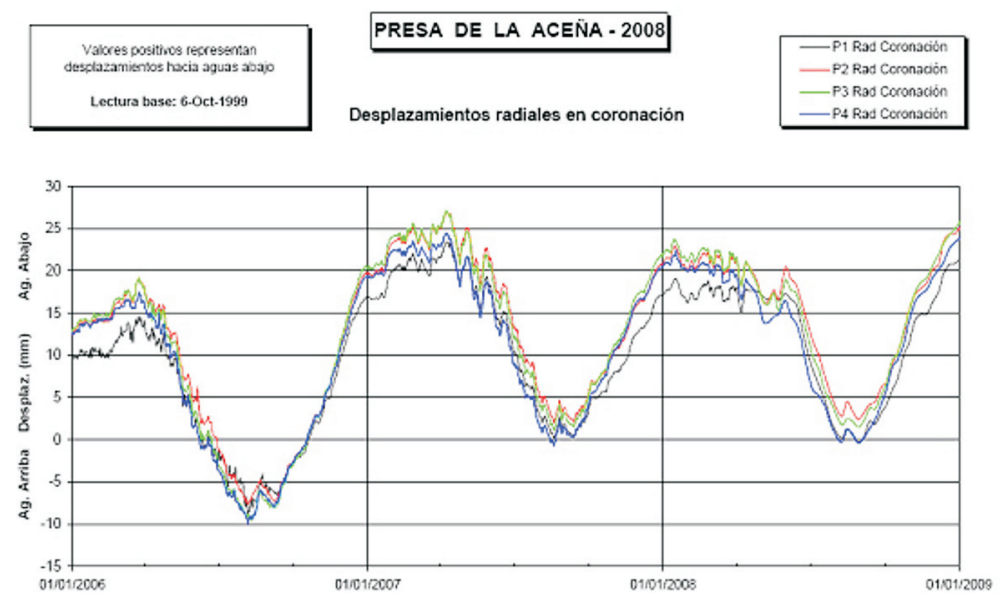

galería horizontal están instalados sendos lectores automáticos de desplazamientos además del dispositivo de lectura manual. Se dispone de una serie continua diaria de datos de los péndulos, (Figura 3).

\subsection{Arquitectura del sistema DGPS}

La red GPS diferencial consta de tres partes fundamentales: Ias antenas y receptores GPS, un sistema de comunicaciones y un sistema informático de proceso de datos.

El sistema de comunicaciones para la transmisión de datos entre los receptores GPS y los servidores fue en un primer momento vía GPRS (General Packet Radio Service), hasta que se completó la instalación de una línea ADSL (Asymmetric Digital Subscriber Line), (Figura 4). 
4. Esquema del sistema de auscultación DGPS. Presa de La Aceña. Fuente: (19)

5. Localización de los péndulos y antenas-receptores GPS en planta. Fuente: (19).

6. Configuración en planta del sistema. Ubicación de antenas y receptores GPS. Fuente: (19).
El software empleado ha sido el 3DTracker (Applied Geomechanics, (20)). Es un programa que ha sido específicamente diseñado para controlar el movimiento preciso y la vigilancia en tiempo real utilizando GPS. Puede procesar los registros de varios receptores al mismo tiempo proporcionando un avanzado y sofisticado sistema de detección en tiempo real de movimientos con soluciones filtradas con exactitud milimétrica.

Las mediciones DGPS son filtradas mediante un filtro Kalman, que puede ser calibrado para responder con lentitud y mayor exactitud (es decir, control diario de las deformaciones en un ciclo superior de tiempo) o más rápidamente con menor exactitud de forma instan-
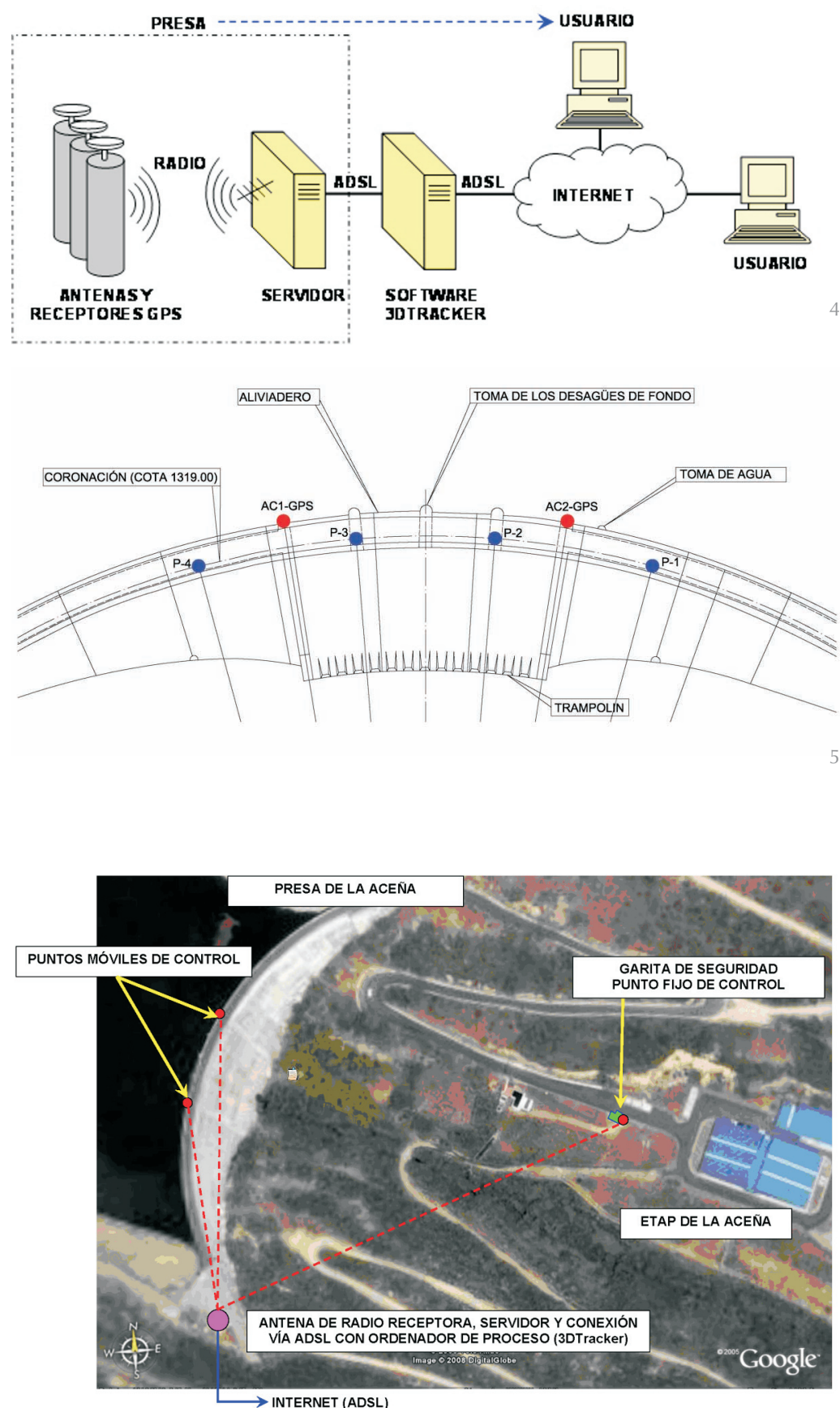

tánea (para registrar por ejemplo, un evento como un asiento o un terremoto, mientras se está produciendo).

\subsection{Configuración espacial}

Se decidió realizar una instalación que permitiese conocer los desplazamientos de dos puntos de la coronación, suficientemente próximos a los péndulos directos de la presa, para correlacionar ambas medidas y comprobar la precisión del sistema. Las antenas y los receptores se instalaron en la coronación de la presa manteniendo la simetría respecto al eje de la misma, (Figura 5).

La estación fija se ubicó aguas abajo de la presa en su margen izquierda, alejada de la misma para asegurar su estabilidad e inmovilidad, (Figura 6).

\subsection{Análisis de resultados}

En primer lugar es necesario exponer las consideraciones realizadas para la configuración del sistema:

- La instalación de la línea ADSL mejoró la conexión, que se veía limitada en la primera fase por la disponibilidad de señal GPRS.

- Al estar la presa ubicada en una zona de montaña, las tormentas afectaron en varias ocasiones sobre los sistemas de alimentación eléctrica del sistema.

A continuación se muestran los resultados comparativos entre las dos técnicas de auscultación, (Figuras 7 y 8 ).

- La comparación de los resultados obtenidos con los péndulos directos (medida considerada verdadera) es buena, con precisiones del orden de $\pm 3 \mathrm{~mm}$, (Figura 7).

- La discontinuidad de datos ocasionada por los cortes de las comunicaciones (Sector-1, Figura 8), afectan a la precisión del sistema puesto que el algoritmo de cálculo del filtro Kalman tarda en estabilizarse.

- Las constantes de configuración del Filtro "Kalman" varían fundamentalmente, con la velocidad de deformación o movimiento de la infraestructura a controlar. Estas constantes se han modificado durante el estudio, con efectos significativos, (Sector-2, Figura 8). Además al ser unas constantes calibradas en base a la velocidad de deformación de la estructura, cuando ésta última es superior a lo normal, la solución filtrada tarda en converger a la medida real (Sector-3, Figura 8). Analizando en detalle el fenómeno denominado "Sector-3", se llega 
a la conclusión de que el rápido descenso de la temperatura ambiente, provocó un punto de inflexión en el gráfico del desplazamiento radial de los péndulos, mientras que el DGPS mostró una discontinuidad debido a la inercia introducida por el filtro. A continuación se produjo un periodo de temperaturas más estables y elevadas, en el que de nuevo, al estabilizarse las velocidades de deformación, el sistema con su modalidad de filtrado, volvió a proporcionar posiciones muy similares a las radiales pendulares. La elección de los parámetros del filtro será uno de los aspectos más importantes a investigar en el futuro en función de los movimientos predecibles en cada estructura.

\section{CONCLUSIONES: VIABILIDAD Y FIABILIDAD}

Se comentan finalmente, algunos aspectos importantes que confirman la viabilidad y fiabilidad de la técnica DGPS en el control de movimientos en grandes estructuras, teniendo en cuenta tanto los la literatura revisada, como los primeros resultados del estudio de caso en la presa de "La Aceña".

Hasta la fecha los resultados son satisfactorios, alcanzándose exactitudes en tiemporeal cercanas a los $\pm 2-3 \mathrm{~mm}$. Además, la amplitud anual de los movimientos dada por los péndulos es muy similar a la obtenida por el sistema DGPS.

Una de las ventajas más notables de la técnica DGPS, y que la hace técnicamente viable en la aplicación para el control de movimientos en estructuras, es la facilidad de montaje, incluso en puntos no accesibles, y la sencillez de operación del sistema.

Por otro lado, es perfectamente compatible con cualquier otro dispositivo de control de movimientos preexistente y el coste asociado es bajo en comparación con otros sistemas de auscultación tradicionales.

Los resultados obtenidos se pueden gestionar en tiempo real con telecontrol y monitorización, lo que influye positivamente en la capacidad de reacción en la gestión de la seguridad de las presas o de cualquier otra infraestructura, como por ejemplo el establecimiento de un sistema de alarma en tiempo real.

Los parámetros de calibración del filtro Kalman influyen, como se ha podido comprobar, en la sensibilidad de la solución del sistema. El post-proceso de los ficheros RINEX (Receiver INdependent Exchange), permitirá depurar los datos obtenidos e identificar el efecto de variables asociadas al sistema, así como comprobar con el postproceso de todos los datos almacenados, la fiabilidad del sistema en tiempo real.

Se puede afirmar que el sistema es útil de cara a la gestión de la auscultación de estructuras como las presas esbeltas, al reflejar adecuadamente el movimiento y la deformación de la misma, con un método alternativo en ambos campos.

Otra ventaja importante es que existe continuidad en las medidas y por tanto del control de la estructura, no como en los métodos topográficos convencionales en los que sólo se puede analizar el comportamiento de la estructura a lo largo de diferentes campañas puntuales.
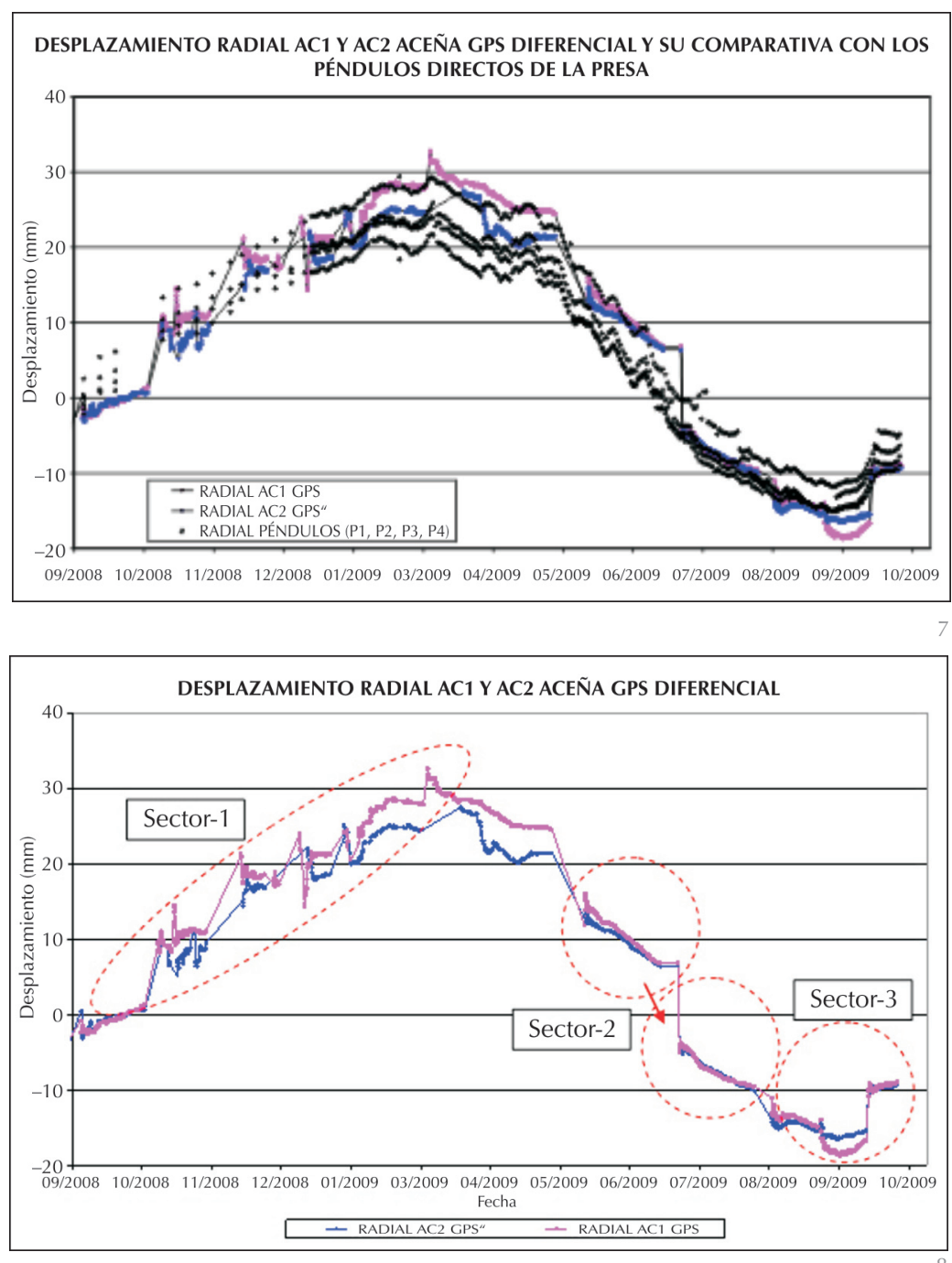

El empleo de la técnica DGPS en grandes estructuras puede proporcionar información sobre las características dinámicas de las mismas, movimientos y deformaciones, frecuencia de vibración, respuestas no lineales durante la vibración,..., gracias a su precisión y frecuencia de toma de datos. Todo esto puede ser analizado en tiempo real, con sistemas automáticos de medición, y alarmas 
programadas para distintos umbrales. Por todo ello se considera que es una técnica fundamental para la auscultación de grandes estructuras, que puede integrarse con otros métodos geotécnicos o topográficos, para la auscultación en la fase constructiva y el seguimiento en la fase de operación.

\section{AGRADECIMIENTOS}

A Ricardo Cifres Giménez por la información, asesoría e instalación DGPS en el estudio de caso.

A Juan Pablo de Francisco (Jefe del Departamento de presas del Canal de Isabel II) y Francisco Blázquez Prieto (Jefe de la División de Seguridad de Presas del CYII) por su total disponibilidad para el desarrollo y aplicación de esta técnica en la presa de "La Aceña".

Esta investigación ha sido realizada en el marco del proyecto SISTEMA INTEGRADO DE AUSCULTACIÓN DE INFRAESTRUCTURAS HIDRÁULICAS EN TIEMPO REAL CON TÉCNICAS GPS: MODELIZACIÓN E INTERPRETACIÓN DE RESULTADOS EN UN CASO REAL del Plan Nacional de I+D+i, 2008.

Este documento constituye un avance de la línea de investigación realizada en el Laboratorio de Topografía y Geomática de la E.T.S.I. de Caminos, Canales y Puertos de Madrid sobre DGPS aplicado a la ingeniería civil y la construcción.

\section{BIBLIOGRAFÍA}

(1) Sillerico, E.; Marchamalo, M.; Rejas, J.G. y Martínez, R.: La técnica DInSAR: bases y aplicación a la medición de subsidencias del terreno en la construcción. Informes de la Construcción 62 (519): 47-53. ISSN 0020-0883. doi: 10.3989/ic.09.0632010.

(2) Olalla Marañón, C.: "Auscultación de laderas", 2007, Jornadas técnicas sobre estabilidad de laderas en embalses, Confederación Hidrográfica del Ebro, Zaragoza. Junio 2007.

(3) Leica Geosystems. http://www.leica-geosystems.es/; http://www.leica-geosystems.es/es/Auscultacionen_1690.htm; http://www.leica-si.com/auscultacion.asp.

(4) IDS. "IBIS-S: Ground-Based Radar Interferometer. An Innovative Technique for Full Scale Static and and Dynamic Structural Monitoring". Dynamic Structural Monitoring http:/www.idscompany.it/

(5) Galán, D.; Martínez Marín, R.; Marchamalo, M.; Sánchez Sobrino, J.A.: CONTROL DE MOVIMIENTOS EN PRESAS MEDIANTE DGPS. APLICACIÓN A LA PRESA DE LA ACEÑA (ESPAÑA). Tecnología y Ciencias del Agua vol. II núm. 3. 2011 (en prensa)

(6) Roberts, G.W.; Meng, X.; Cosser, E.; Dodson, A.H.: "The Use of Single Frequency GPS to Measure the Deformations and Deflections of Structures". FIG Working Week 2004 Athens, Grecia, Mayo 22-27, 2004.

(7) Cheng Penggen; Shi Wenzhong; y Zheng Wanxing: "Large Structure Health Dynamic Monitoring Using GPS Technology". Engineering Surveys for Construction Works and Structural Engineering. FIG XXII International Congress. Washington, D.C. USA, Abril 19-26 2002

(8) Psimoulis, P.A.; Psimoulis, P.A.; Pytharouli, P.; Stiros, S. 2006: "Experimental monitoring of oscillations of major flexible structures using GPS and RTS". 3rd IAG / 12th FIG Symposium, Baden (Austria), Mayo 22-24, 2006

(9) Kalman, R.E.: "A New Approach to Linear Filtering and Prediction Problems" Transaction of the ASMEJournal of Basic Engineering, pp. 35-45 (March 1960).

(10) Welch, G.; Bishop, G.: "An Introduction to the Kalman Filter", TR 95-041, Department of Computer Science, University of North Carolina. USA. 81 pp. 2002

(11) Solera Ramírez, A.: "El filtro de Kalman", Banco central de Costa Rica, Dpto. de investigaciones económicas. 33 pp. 2003.

(12) Hofmann-Wellenhof, B.; Lichtenegger, H.; Collins, J.: Springer-Verlag Wien NewYork, "GPS Theory and Practice" 326 pp. 2001

(13) GOCA. 2002. IllwerkeVorarlberg, Austria, GOCA-Project-Examples, "Monitoring of Kops Dam", GPS-based online Control and Alarm System. http:www.goca.info/beispiel_e.html.

(14) Behr, J.A.; Hudnut, K.W.; King, Nancy E.: "Monitoring Structural Deformation at Pacoima Dam, California Using Continuous GPS", Southern California Earthquake Center, SCIGN-USGS, EEUU, United States Geological Survey. 10 pp. 2000

(15) Wübbena, G.; Bagge, A.: Boettcher, G.; Schmitz. M.: "Permanent ObjectMonitoring with GPS with 1 MillimeterAccuracy". International Technical Meeting, ION GPS-01, September 11.-14.; 2001, Salt Lake City, Utah. 2001.

(16) Çelebi, M.: "GPS in dinamic monitoring of long-period structures". Soil Dynamics and Earthquake Engineering. Volume 20, Issues 5-8, December 2000, Pages 477-483. doi:10.1016/S0267-7261(00)00094-4. 2000

(17) Rutledge, D.R.; Meyerholtz, S.Z.: "Performance monitoring of Libby Dam with a differential global positioning system" 25th United States Society on Dams (USSD) Annual Meeting. Salt Lake City, Utah, USA. Junio 6-10,2005

(18) Solanes Pardo, F.; Calderón, P.A.; Escuder, I.; Martínez Ortega, J.: "Estabilización de la ladera de Puebla de Arenós en el Embalse de Arenós, Castellón", Jornadas técnicas sobre estabilidad de laderas en embalses, Confederación Hidrográfica del Ebro, Zaragoza junio 2007.

(19) Blázquez F.; Galán D.: "Informe anual de auscultación Presa de La Aceña-Año 2008". División Seguridad de Presas. Canal de Isabel II. Madrid. 2009,

(20) Applied Geomechanics. 3Dtracker Software. USA. http://www.geomechanics.com/ 